\title{
COMUNICACIÓN
}

\section{ELABORACIÓN DE BLOQUES NUTRICIONALES Y EVALUACIÓN DE LA RESISTENCIA}

\author{
Manuel Rios A. ${ }^{1}$, Felipe San Martín H. ${ }^{2}$ y Fernando Carcelén C.
}

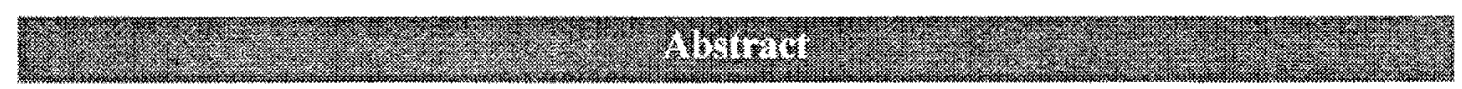

Nutritional blocks containing $10 \%$ urea, $10 \%$ calcium phosphate, $7.5 \%$ calcium oxide, $2.5 \%$ calcium sulfate, $10 \%$ bran, and $10 \%$ cotton paste, were modified by addition of the following ingredients: Formula $1-25 \%$ bentonite, $15 \%$ molasses and $10 \%$ salt; Formula $2-40 \%$ sodium bentonite and $10 \%$ salt; Formula $3-30 \%$ sodium bentonite and $20 \%$ salt; and Formula $4-50 \%$ salt. The resistance of each of the four blocks was measured. Formulas 1, 2 and 3 averaged an acceptable $86.5 \mathrm{~kg} / \mathrm{cm}^{2}(\mathrm{P}<0.01)$, while Formula 4 was unacceptable at $\left(19.1 \mathrm{~kg} / \mathrm{cm}^{2}\right)$.

Key words: Nutritional block, resistance.

Palabras Clave: Bloque nutricional, resistencia.

En la actividad ganadera, constantemente estamos recurriendo al uso de suplementación alimenticia de diferentes tipos para diferentes especies de animales, estados fisiológicos, niveles productivos y estados de salud. La suplementación tiene como objetivo cubrir parte de los requerimientos que no cubre la dieta base a través del uso de un alimento o conjunto de alimentos que denominamos suplementos, para corregir fallas en la producción.

El bloque nutricional es un material alimenticio sólido, que provee constante y lentamente nutrientes al animal. Pueden ser elaborados con una tecnología económica artesanal o semiartesanal con varios subproductos locales que se pueden adaptar a las condiciones variables en que operan los productores.

\footnotetext{
Practica privada

${ }^{z}$ Laboratorio de Nutrición - FMV - UNMSM. Email: d170032@unmsm.edupe
}

Diversos autores señalan a la dureza o resistencia $\left(\mathrm{kg} / \mathrm{cm}^{2}\right)$ del bloque como el factor principal para el consumo mediante lamidas o mordidas, permitiendo ingerir cantidades apropiadas. Bloques muy duros o muy blandos determinan que el animal no reciba la suplementación nutricional adecuada. Por otro lado la dureza da calidad al bloque permitiendo el transporte y el almacenamiento. (Sansoucy 1986; Birbe et al., 1994a).

La dureza o resistencia del bloque nutricional (aspecto físico) depende básicamente del tipo y cantidad de materia prima, tipo y cantidad de aglomerante (cal, bentonita sódica, cemento, melaza, etc.), tiempo de secado y de la energía de compactación aplicada sobre la masa $\left(\mathrm{kg} / \mathrm{cm}^{2}\right)$. (Sansoucy, 1987; Birbe et al;1994b). A medida que se aumenta la energía de compactación sobre el bloque aumenta la resistencia.

La tecnología de fabricación de los bloques nutricionales ha sido estudiada por distintos investigadores. Sin embargo algunos factores físicos como la dureza que pudiera 
influenciar en el aprovechamiento de los bloques no han sido evaluados. Esta comunicación informa sobre un estudio de resistencia o dureza de bloques al final del secado. Se trabajó con cuatro formulas (Cuadro) l) donde se modificó los niveles de sal. bentonita! melaza. horas de secado y tipo de recido (ertufa! medio ambiente).

Cuadro 1. Composición (\%) de los bloques nutricionales.

\begin{tabular}{|c|c|c|c|c|}
\hline \multirow{2}{*}{ Ingredientes } & \multicolumn{4}{|c|}{ Bloque nutricional } \\
\hline & Melaza & Bentonita 40 & Bentonita 30 & Sal 50 \\
\hline Sales minerales & 10.0 & 10.0 & 20.0 & 50.0 \\
\hline Urea & 10.0 & 10.0 & 10.0 & 10.0 \\
\hline Fosfato de Calcio & 10.0 & 10.0 & 10.0 & 10.0 \\
\hline Oxido de Calcio & 7.5 & 7.5 & 7.5 & 7.5 \\
\hline Sulfato de Calcio & 2.5 & 2.5 & 2.5 & 2.5 \\
\hline Bentonita sódica & 25.0 & 40.0 & 30.0 & $\cdots--$ \\
\hline Afrecho & 10.0 & 10.0 & 10.0 & 10.0 \\
\hline Pasta de algodón & 10.0 & 10.0 & 10.0 & 10.0 \\
\hline Melaza & 15.0 & $-\cdots-$ & $-\cdots--$ & $-\cdots--$ \\
\hline Total & $100 \%$ & $100 \%$ & $100 \%$ & $100 \%$ \\
\hline $\begin{array}{l}\text { Energía de compactación } \\
\text { aplicada al bloque }\left(\mathrm{kg} / \mathrm{cm}^{2}\right)\end{array}$ & 5.15 & 5.15 & 5.15 & 6.43 \\
\hline
\end{tabular}

La elaboración de los bloques nutricionales y posterior evaluación sé realizaron en el Laboratorio de Bioquímica. Nutrición y Alimentación Animal de la Facultad de Medicina Veterinaria, durante los meses de verano.

Los equipos empleados fucron: espátula, bateas de plástico. tarimas de madera. balanza de precisión. estufa y prensa compactadora. Para su elaboración se pesaron los ingredientes segín el porcentaje señalado en cada fómula, se mezcló completamente la masa y luego fue introducida en el molde para ser compactado.

Se elaboraron 64 bloques nutricionales, 32 fueron secados en estufa a $60^{\circ} \mathrm{C}$ y 32 fueron secados a medio ambiente a $26^{\circ} \mathrm{C}$ promedio, correspondiendo a dos bloques de cada fórmula por cada tiempo de secado $(48,72$, $96,120 \mathrm{~h}$ ). Otro grupo de 64 bloques con 4 repeticiones para cada fórmula y para cada tiempo de secado (1, 2. 3, 4 sem.), se secó al medio ambiente. Cumplido el tiempo de $x$ cado se registró la resistencia a la penctratción $\mathrm{kg} / \mathrm{cm}^{2}$, la cual fue medida mediante un penetrómetro de bolsillo tomando como base las normas de evaluación usadar en fisica de sut los y resistencia de materiales (Birbe al al., 1994a: Birbe 't al.1994b: Ortiz? Baumeister. 1994).

Los resultados de resistencia a la penetración fueron evaluados por A.VDI: V.I! los promedios de las variables independientes (bloque nutricional y tiempo) se analizatron mediante la prueba de Duncan ( $p-0.05)$. Estos análisis fueron realizados con el sofiware SAS (Stadistical Analy sis System).

En el Cuadro 2 se aprecia que enl las fórmulas bentonita 40 ? bentonita 30. la rissistencia aumenta conforme pasan las horas de secado. En las otras förmular la resistencia es menor debido a sucomposicion. le yuc coincide con Arts et al. 1990. 
Cuadro 2. Resistencia a la penetración $\left(\mathrm{kg} / \mathrm{cm}^{2}\right)$ de bloques nutricionales secados a estufa $\left(60^{\circ}\right)$ y a medio ambiente $\left(26^{\circ} \mathrm{C}\right)$.

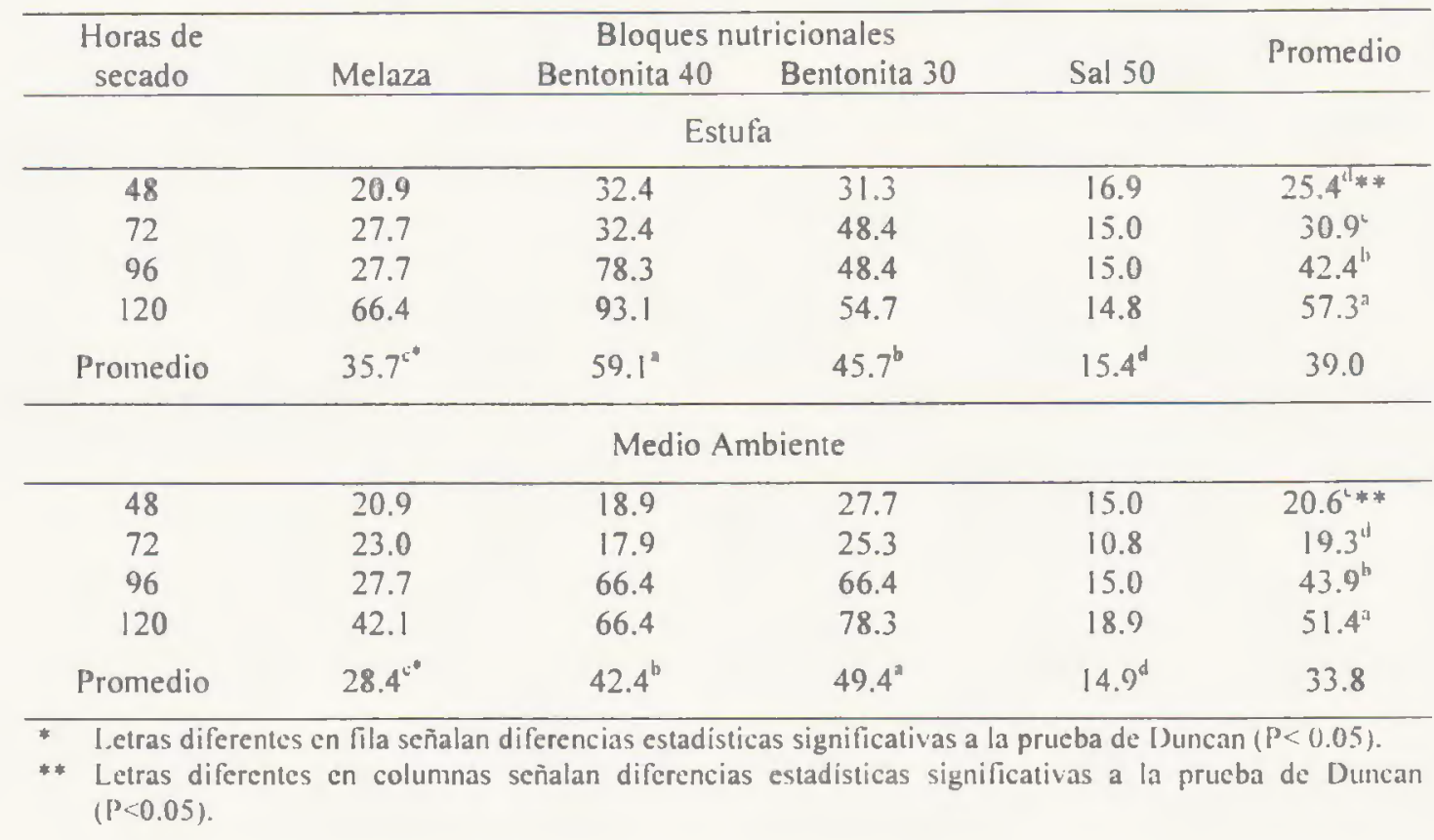

Después de cuatro horas de secado al medio ambiente (Cuadro 3 ) las fórmulas bentonita 40 , bentonita 30 y melaza presentaron una resistencia adecuada con promedio de $86.5 \mathrm{~kg} / \mathrm{cm}^{2}(P<0.01)$ superior a lo obtenido con los bloques nutricionales de la fórmula sal $50\left(19.1 \mathrm{~kg} / \mathrm{cm}^{2}\right)$ concluyéndose que el uso de $30-40 \%$ de bentonita sódica y $15 \%$ de melaza permite obtener bloques de excelente calidad después de la segunda semana de secado, mientras que bloques con altos porcentajes de sales minerales $(50 \%)$ o urea necesitan mayor proporción de aglomerante (25 - 40\%). Además la energia de compactación optima es $5 \mathrm{~kg} / \mathrm{cm}^{2}$ o más siempre y cuando la fórmula tenga porcentaje adecuado de aglomerante.

Cuadro 3. Resistencia a la penetración $\left(\mathrm{kg} / \mathrm{cm}^{2}\right)$ de los bloques nutricionales secados al medio ambiente a $26^{\circ} \mathrm{C}$.

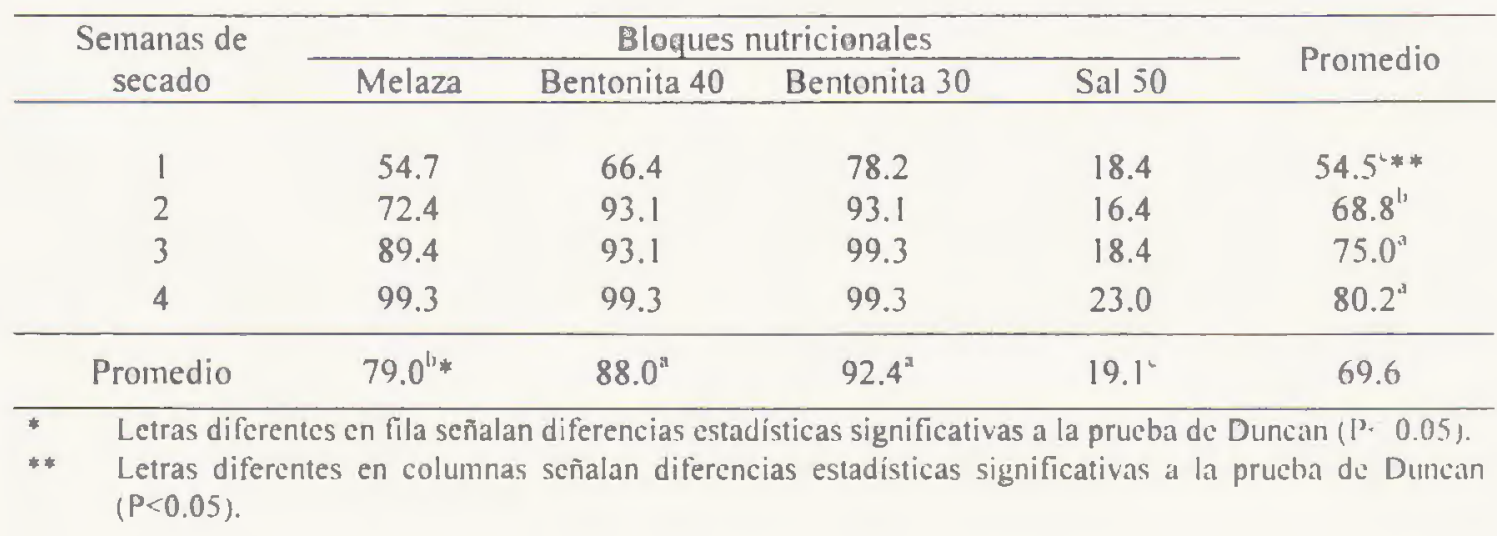




\section{Literatura Citada}

1. Aarts G, D. Sansoucy y G. Levieux. 1990. Guideline for the manufacture service. Animal production and Health. División FAD consultant. Rome - Italy $19 \mathrm{p}$.

2. Birbe B, P. Herrera y' D. Mata. 1994a. Bloques Nutricionales: una alternativa en ganadería de doble propósito. Universidad Nacional Experimental Simón Rodriguez - Universidad Nacional Rómulo Gallegos. 33p.

3. Birbe B, E. Chacoin, L. Tay/hardat, J. Garmendis y D. Mata. 1994b. Aspectos físicos de importancia en la fabricación y utilización de bloques multinutricionales. Folleto de bloques
Multinutricionales. In: Cardozo FA, Birbe B, eds. UNELLES. p.: $1-14$.

4. Ortiz P.R. y A.A. Baumeister. 1994. Consideraciones en la preparación y uso de los bloques nutricionales. Folleto del curso sobre Bloques Multinutricionales. In: Cardozo FA, Birbe B, eds. LNELLES. p.:85-90.

5. Sansoucy R. 1986. The Sahel Manufacture of Melasses - urea blocks. World Animal Review. 57 . p.: $40-48$.

6. Sansoucy R. 1987. Los bloques melaza - Urea como Suplemento Multinutriente para rumiantes. Taller Internacional de la Fundación Internacional para la ciencia sobre la Melaza, como recurso alimenticio para la producción animal. Universidad de Camagüey. Cuba. 13 - 18 julio. p.:16. 